PROCEEDINGS OF THE AMERICAN MATHEMATICAL SOCIETY

Volume 124, Number 5, May 1996

\title{
EIGENVALUE RATIOS FOR THE REGULAR STURM-LIOUVILLE SYSTEM
}

\author{
YU-LING HUANG AND C. K. LAW
}

(Communicated by Hal L. Smith)

\begin{abstract}
Following the method of Ashbaugh-Benguria in Comm. Math. Phys. 124 (1989), 403-415; J. Differential Equations 103 (1993), 205-219, we prove an upper estimate of the arbitrary eigenvalue ratio $\left(\mu_{m} / \mu_{n}\right)$ for the regular Sturm-Liouville system. This upper estimate is sharp for Neumann boundary conditions. We also discuss the sign of $\mu_{1}$ and include an elementary proof of a useful trigonometric inequality first given in the aforementioned articles.
\end{abstract}

\section{INTRODUCTION}

A regular Sturm-Liouville system on $(0,1)$ [4, p.300] is the regular SturmLiouville equation:

$$
-\left(p(x) y^{\prime}\right)^{\prime}+q(x) y=\mu w(x) y,
$$

where

$$
p \in C^{1}[0,1], \quad q, w \in C[0,1], \quad p, w>0 ;
$$

with separated endpoint boundary conditions

$$
\left\{\begin{array}{l}
y(0) \cos \gamma-p(0) y^{\prime}(0) \sin \gamma=0 \\
y(1) \cos \delta-p(1) y^{\prime}(1) \sin \delta=0
\end{array}\right.
$$

where $0 \leq \gamma<\pi, 0<\delta \leq \pi . \gamma$ and $\delta$ are in fact the initial and final phases respectively in the classical Prüfer substitution.

It is well known that the above system has an increasing sequence of simple real eigenvalues

$$
\mu_{1}<\mu_{2}<\cdots<\mu_{n}<\mu_{n+1}<\cdots,
$$

and the eigenfunction corresponding to $\mu_{n}$ has exactly $n$ zeros in $(0,1)$. Sometimes, an additional eigenvalue $\mu_{0}<\mu_{1}$ may also occur, and the corresponding eigenfunction has no zero in $(0,1)$.

Received by the editors July 6, 1994 .

1991 Mathematics Subject Classification. Primary 34B24, 34L15.

Key words and phrases. Regular Sturm-Liouville system, Neumann boundary conditions, eigenvalue ratio, modified Prüfer substitution.

This research is partially supported by the National Science Council, Taiwan, R. O. C. under contract number NSC-83-0208-M-110-028.

(C)1996 American Mathematical Society 
Recently, Ashbaugh and Benguria [3] proved an optimal upper estimate of the eigenvalue ratio $\lambda_{m} / \lambda_{n}(m>n \geq 1)$ for the Dirichlet problem when $\gamma=0$ and $\delta=\pi$, with the assumptions that

$$
q \geq 0 \quad \text { and } \quad 0<k \leq p w \leq K .
$$

Based on a modified Prüfer substitution and trigonometric inequality, they proved that

$$
\frac{\lambda_{m}}{\lambda_{n}} \leq\left(\left\lceil\frac{m}{n}\right\rceil\right)^{2} \frac{K}{k}
$$

with equality when and only when $q \equiv 0$ and $k=K$. This is a generalization of their previous work on one-dimensional Schrödinger operators [1, 2]. (In this paper, we adopt the following notation. Let $\lceil s\rceil$ denote the value of the ceiling function at $s$, i.e. $\lceil s\rceil=\min \{n \in \mathbf{N}, n \geq s\}$, and $\lfloor s\rfloor$ denote the value of the floor function at $s,\lfloor s\rfloor=\max \{n \in \mathbf{N}, n \leq s\}$.)

We extend their result to the general regular Sturm-Liouville system. The main idea is to allow the modified phase to take up a negative initial value and in the induction process we divide the interval $[0,1]$ into two parts by the point where the phase first attains $\pi / 2$. Our main theorem is

Theorem 1. For the regular Sturm-Liouville system (1)-(3), if $q(x) \geq 0$ and $0<k \leq p w \leq K$, then for any integers $m>n \geq 1$ discussed below, the eigenvalues $\mu_{m}$ and $\mu_{n}$ are positive, and

$$
\frac{\mu_{m}}{\mu_{n}} \leq l^{2} \frac{K}{k}
$$

where $l$ is determined by the boundary conditions as follows:

\begin{tabular}{l|ll} 
& $\pi \geq \pi-\gamma \geq \pi / 2$ & $\pi / 2>\pi-\gamma>0$ \\
\hline$\pi \geq \delta \geq \pi / 2$ & $2\left(\left\lfloor\frac{1}{2}\left\lceil\frac{m}{n}\right\rceil\right\rfloor\right)+1$ & $2\left\lceil\frac{m}{n}\right\rceil-1$ \\
$\pi / 2>\delta>0$ & $2\left\lceil\frac{m}{n}\right\rceil-1$ & $2\left\lceil\frac{m}{n}\right\rceil-1($ when $n>1)$
\end{tabular}

The above theorem has an interesting corollary on the Neumann boundary conditions. The inequality is sharp there!

Theorem 2. For the regular Sturm-Liouville system (1) and (2) with Neumann boundary conditions $(\delta=\gamma=\pi / 2)$, if $q \geq 0$ and $0<k \leq p w \leq K$, then for any integers $m>n \geq 1$,

$$
\frac{\mu_{m}}{\mu_{n}} \leq\left\{2\left(\left\lfloor\frac{1}{2}\left\lceil\frac{m}{n}\right\rceil\right\rfloor\right)+1\right\}^{2} \frac{K}{k}
$$

with equality when and only when $m$ is an odd multiple of $n, q \equiv 0$, and $k=K$.

We note that for any integer $l$, the function

$$
2\left(\left\lfloor\frac{l}{2}\right\rfloor\right)+1= \begin{cases}l & \text { when } l \text { is odd, } \\ l+1 & \text { when } l \text { is even, }\end{cases}
$$

so that $\lceil m / n\rceil$ is odd when and only when

$$
2\left(\left\lfloor\frac{1}{2}\left\lceil\frac{m}{n}\right\rceil\right\rfloor\right)+1=\left\lceil\frac{m}{n}\right\rceil .
$$

Thus Theorem 2 is analogous to Ashbaugh-Benguria's result for Dirichlet boundary conditions above.

At the end of this section, we shall give an elementary proof of a trigonometric inequality in $[2,3]$. This inequality is interesting itself and is critical in the method. 
In section 2, we introduce the modified Prüfer substitution and the Comparison Theorem. The function $\tan ^{-1}(s \tan \theta)$ is also analyzed there. The main theorems are proved in section 3. In section 4 , we discuss the sign of $\mu_{1}$ and obtain an upper estimate for $\mu_{m} / \mu_{1}$ for $0<\delta, \pi-\gamma<\pi / 2$ when the potential function $q$ is sufficiently large.

Theorem 3. Let $c>1$. Then for $0<\theta<\lfloor c\rfloor \pi / c$,

$$
|\sin c \theta|<c \sin \theta \text {. }
$$

Proof. When $c$ is integral-valued, the inequality can easily be proved by mathematical induction, using the compound angle formula for the sine function. On the other hand, since $f(x)=\sin x$ is a strictly concave function in $[0, \pi]$, we have for any $\phi \in(0, \pi)$,

$$
\sin \left(\frac{\phi}{c}\right)>\frac{1}{c} \sin \phi
$$

Thus for arbitrary $c \in(1,2)$, let $\phi=c \theta$; the above inequality implies $c \sin \theta>\sin c \theta$. Inductively, suppose the above inequality holds for any $c \in[1, j]$ for $j \geq 2$. Let $c=p+1 \in(j, j+1)$. It is easy to show that $|\sin c \theta|<c \sin \theta$ when $0<p \theta<\lfloor p\rfloor \pi$. And when $\frac{\pi}{2}<\frac{\lfloor p\rfloor \pi}{p} \leq \theta<\frac{\lfloor p\rfloor+1}{p+1} \pi$,

$$
0<(\lfloor p\rfloor+1) \pi-(p+1) \theta \leq \pi-\theta<\frac{\pi}{2} .
$$

Therefore

$$
|\sin (p+1) \theta| \leq \sin \theta<(p+1) \sin \theta .
$$

The proof is complete.

Corollary 4. If $c \geq 1$ and $|\theta| \leq\lfloor c\rfloor \pi / c$, then $\sin ^{2} c \theta \leq c^{2} \sin ^{2} \theta$.

\section{Preliminaries}

We shall apply the modified Prüfer substitution as introduced in [3] to the regular Sturm-Liouville system. Let

$$
\begin{aligned}
y & =r(x) \sin a \sqrt{\mu} \theta, \\
p y^{\prime} & =a \sqrt{\mu} r(x) \cos a \sqrt{\mu} \theta .
\end{aligned}
$$

Then

$$
r^{2}=y^{2}+\frac{\left(p y^{\prime}\right)^{2}}{a^{2} \mu} \quad \text { and } \quad \tan a \sqrt{\mu} \theta=\frac{a \sqrt{\mu} y}{p y^{\prime}} .
$$

Also from (1), we derive

$$
\begin{aligned}
\frac{d \theta}{d x} & =\frac{1}{p}-\left(\frac{1}{p}-\frac{w}{a^{2}}\right) \sin ^{2} a \sqrt{\mu} \theta-\frac{q}{a^{2} \mu} \sin ^{2} a \sqrt{\mu} \theta \\
& =\Phi_{(a, \mu)}(x, \theta) .
\end{aligned}
$$

Eq. (4) represents different first-order equations for different values of $a$ and $\mu$. Moreover, suppose $y_{m}$ is the eigenfunction associated with the eigenvalue $\mu_{m}$; the corresponding Eq. (4) has boundary values

$$
\left\{\begin{array}{lll}
a \sqrt{\mu_{m}} \theta(0) & =\tan ^{-1}\left(a \sqrt{\mu_{m}} \tan \gamma\right), & 0 \leq \gamma<\pi \\
a \sqrt{\mu_{m}} \theta(1) & =\tan ^{-1}\left(a \sqrt{\mu_{m}} \tan \delta\right)+m \pi, & 0<\delta \leq \pi
\end{array}\right.
$$


The inverse function $\tan ^{-1}$ takes value in $[0, \pi]$ instead of the usual $\left(-\frac{\pi}{2}, \frac{\pi}{2}\right)$. In particular, let $\tan ^{-1}(a \sqrt{\mu} \tan \phi)=\phi$ where $\phi=0, \frac{\pi}{2}, \pi$.

Lemma 5. Fix any $\gamma \in[0, \pi)$; the function $f$ defined as

$$
f_{\gamma}(s)=\frac{1}{s}\left[\tan ^{-1}(s \tan \gamma)-\pi\right]
$$

is strictly increasing in $s \in \mathbf{R}^{+}$.

Proof. First $f_{0}(s)=-\frac{\pi}{s}$ and $f_{\frac{\pi}{2}}(s)=-\frac{\pi}{2 s}$. Both functions are strictly increasing. For the other $f_{\gamma}$ 's,

$$
f_{\gamma}^{\prime}(s)=\frac{s \tan \gamma-\left[\tan ^{-1}(s \tan \gamma)-\pi\right]\left(1+s^{2} \tan ^{2} \gamma\right)}{s^{2}\left(1+s^{2} \tan ^{2} \gamma\right)}
$$

Let $\varphi=\tan ^{-1}(s \tan \gamma) \in[0, \pi)$. Then the numerator of $f_{\gamma}^{\prime}$ is given by

$$
u(\varphi)=\tan \varphi+(\pi-\varphi)\left(1+\tan ^{2} \varphi\right) .
$$

Since $u$ is positive for $0<\gamma<\frac{\pi}{2}$, the corresponding $f_{\gamma}$ is strictly increasing. When $\frac{\pi}{2}<\gamma<\pi$, then $\frac{\pi}{2}<\varphi<\pi$ and $\tan \varphi<0$, and

$$
u^{\prime}(\varphi)=2(\pi-\varphi) \tan \varphi \sec ^{2} \varphi<0 .
$$

Since $u(\pi)=0$, it follows that $u(\varphi)>0$ for $\frac{\pi}{2}<\varphi<\pi$. Therefore $f_{\gamma}^{\prime}>0$ and $f_{\gamma}$ is strictly increasing.

Corollary 6. For any $\varphi \in[0, \pi]$ and $j \in \mathbf{N}$, if $1 \leq s \leq j$, then

$$
\tan ^{-1}(s \tan \varphi)+(j-1) \pi \geq j \varphi .
$$

Proof. It is evident that the inequality holds when $\varphi=\pi$. When $\varphi \in[0, \pi)$, we have from Lemma 5 that $\tan ^{-1}(s \tan \varphi)-\pi \geq s(\varphi-\pi)$. Hence

$$
\tan ^{-1}(s \tan \varphi)-\varphi \geq(s-1)(\varphi-\pi) \text {. }
$$

And since $1 \leq s \leq j$,

$$
\tan ^{-1}(s \tan \varphi)-\varphi \geq(j-1)(\varphi-\pi)
$$

Lemma 7. For any $j \in \mathbf{N}, c \in \mathbf{R}$, define the function

$$
g(\varphi)=\frac{1}{\varphi}\left\{j \pi+\tan ^{-1}(c \tan \varphi)\right\}
$$

for $\varphi \in(0, \pi)$. If $1<c \leq g(\varphi)$, then $g^{\prime}(\varphi)<0$. Hence if $1<c \leq g\left(\varphi_{0}\right)$ for some $\varphi_{0} \in(0, \pi)$, then for any $0<\varphi<\varphi_{0}, c \leq g\left(\varphi_{0}\right)<g(\varphi)$.

Proof. For $\varphi \in\left(0, \frac{\pi}{2}\right) \cup\left(\frac{\pi}{2}, \pi\right]$,

$$
\begin{aligned}
g^{\prime}(\varphi) & =\frac{-1}{\varphi^{2}}\left[j \pi+\tan ^{-1}(c \tan \varphi)\right]+\frac{1}{\varphi}\left[\frac{c \sec ^{2} \varphi}{1+c^{2} \tan ^{2} \varphi}\right] \\
& <\frac{1}{\varphi}\left[c-\frac{1}{\varphi}\left(j \pi+\tan ^{-1}(c \tan \varphi)\right)\right] \\
& \leq 0
\end{aligned}
$$


Also, by L'Hôpital's rule,

$$
\begin{aligned}
g^{\prime}\left(\frac{\pi}{2}\right) & =\lim _{\phi \rightarrow \pi / 2} \frac{j \pi+\tan ^{-1}(c \tan \phi)-(2 j+1) \phi}{\phi(\phi-\pi / 2)} \\
& =-\frac{2(2 j+1)}{\pi}+\frac{2}{\pi} \lim _{\phi \rightarrow \pi / 2} \frac{\tan ^{-1}(c \tan \phi)-\pi / 2}{\phi-\pi / 2} \\
& =\frac{2}{c \pi}[1-(2 j+1) c] \\
& <0 .
\end{aligned}
$$

The modified Prüfer substitution turns the problem into a first order differential equation. Hence we can use a comparison theorem to compare the growth of solutions for two different first order initial value problems. We state the following modified version of $[4$, p.29] for future use.

Comparison Theorem. Consider two differential equations on $[0,1]$,

$$
\begin{aligned}
& \theta_{1}^{\prime}(x)=F\left(x, \theta_{1}(x)\right), \\
& \theta_{2}^{\prime}(x)=G\left(x, \theta_{2}(x)\right) .
\end{aligned}
$$

Suppose $F$ or $G$ is Lipschitz in $\theta$, and $F(x, \theta) \leq G(x, \theta)$ on $[0,1] \times I$ for some interval I. If $\theta_{1}(0) \leq \theta_{2}(0)$ and $\theta_{2}(x)$ lies in the interior of $I$ for every $x$ in $(0,1)$, then $\theta_{1} \leq \theta_{2}$ on $[0,1]$. In fact, take any $x_{0} \in[0,1]$, either $\theta_{1}\left(x_{0}\right)<\theta_{2}\left(x_{0}\right)$ or $\theta_{1}=\theta_{2}$ on $\left[0, x_{0}\right]$.

\section{Proof of MAIN THEOREMS}

Theorem 8. For the regular Sturm-Liouville system (1)-(3), if $q \geq 0,0<k \leq$ $p w \leq K$ and $\mu_{1}>0$, then for any integer $m \geq 1$,

$$
\frac{\mu_{m}}{\mu_{1}} \leq\left(\frac{\lfloor m / 2\rfloor \pi+\phi_{k \mu_{m}}}{\phi_{K \mu_{1}}}\right)^{2} \frac{K}{k}
$$

where

$$
\phi=\min \{\delta, \pi-\gamma\}, \quad \phi_{s}=\tan ^{-1}(\sqrt{s} \tan \phi) .
$$

$$
\frac{\mu_{m}}{\mu_{1}}<\left(\frac{(m-1) \pi+\varphi_{k \mu_{m}}}{\varphi_{K \mu_{1}}}\right)^{2} \frac{K}{k},
$$

where

$$
\varphi=\max \{\delta, \pi-\gamma\}, \text { and } \varphi_{s}=\tan ^{-1}(\sqrt{s} \tan \varphi) .
$$

Proof. For (a), we actually need to show

$$
\frac{\mu_{m}}{\mu_{1}} \leq\left(\max \left\{\frac{\lfloor m / 2\rfloor \pi+\delta_{k \mu_{m}}}{\delta_{K \mu_{1}}}, \frac{(\lfloor m / 2\rfloor+1) \pi-\gamma_{k \mu_{m}}}{\pi-\gamma_{K \mu_{1}}}\right\}\right)^{2} \frac{K}{k}
$$

$\left(\gamma_{s}=\tan ^{-1}(\sqrt{s} \tan \gamma)\right.$ and $\left.\delta_{s}=\tan ^{-1}(\sqrt{s} \tan \delta)\right)$. The two choices arise because the eigenvalues $\mu_{m}$ for (1) are invariant under the transformation $x \longmapsto 1-x$, while the phases at the boundary become $\pi-\delta$ and $\pi-\gamma$. Then Lemma 7 asserts 
that the choice of the maximum is equivalent to the minimum of the alternative phases (i.e. $\min \left\{\delta_{K \mu_{1}}, \pi-\gamma_{K \mu_{1}}\right\}$ ). Hence we may simply assume that

$$
\delta_{K \mu_{1}} \leq \pi-\gamma_{K \mu_{1}} \quad \text { or } \quad \delta \leq \pi-\gamma
$$

and show that

$$
\frac{\mu_{m}}{\mu_{1}} \leq\left(\frac{\lfloor m / 2\rfloor \pi+\delta_{k \mu_{m}}}{\delta_{K \mu_{1}}}\right)^{2} \frac{K}{k}
$$

We compare two different equations of form (4),

$$
\frac{d \theta_{j}}{d x}=F_{j}\left(x, \theta_{j}\right) \quad(j=1, m),
$$

where

$$
\left\{\begin{array}{l}
F_{1}(x, \theta)=\Phi_{\sqrt{K}, \mu_{1}}(x, \theta), \\
F_{m}(x, \theta)=\Phi_{\sqrt{k}, \mu_{m}}(x, \theta) .
\end{array}\right.
$$

We let $\theta_{j}(j=1, m)$ denote the modified phase function of the $j$ th eigenfunction, with initial values

$$
\begin{gathered}
\sqrt{K \mu_{1}} \theta_{1}(0)=\tan ^{-1}\left(\sqrt{K \mu_{1}} \tan \gamma\right)-\pi \\
=\gamma_{K \mu_{1}}-\pi \\
\sqrt{k \mu_{m}} \theta_{m}(0)=\tan ^{-1}\left(\sqrt{k \mu_{m}} \tan \gamma\right)-\left\lfloor\frac{m+1}{2}\right\rfloor \pi \\
=\gamma_{k \mu_{m}}-\left\lfloor\frac{m+1}{2}\right\rfloor \pi .
\end{gathered}
$$

Hence

$$
\theta_{1}(1)=\frac{\delta_{K \mu_{1}}}{\sqrt{K \mu_{1}}}, \quad \theta_{m}(1)=\frac{\lfloor m / 2\rfloor \pi+\delta_{k \mu_{m}}}{\sqrt{k \mu_{m}}}
$$

Assume

$$
\frac{\mu_{m}}{\mu_{1}}>\left(\frac{\lfloor m / 2\rfloor \pi+\delta_{k \mu_{m}}}{\delta_{K \mu_{1}}}\right)^{2} \frac{K}{k} \quad \text { for some } m>1 .
$$

By (7), (8), and (9),

$$
c \equiv \sqrt{\frac{k \mu_{m}}{K \mu_{1}}}>\frac{\left\lfloor\frac{m+1}{2}\right\rfloor \pi-\gamma_{k \mu_{m}}}{\pi-\gamma_{K \mu_{1}}},
$$

and thus $\theta_{1}(0)<\theta_{m}(0)$. Then from $k \mu_{m}>K \mu_{1}$ and Corollary 4,

$$
\frac{\sin ^{2} \sqrt{K \mu_{1}} \theta}{K \mu_{1}} \leq \frac{\sin ^{2} \sqrt{k \mu_{m}} \theta}{k \mu_{m}}
$$

for $\theta \in I=[-A, A], A=\frac{\lfloor c\rfloor \pi}{\sqrt{k \mu_{m}}}$, Hence $F_{1}(x, \theta) \leq F_{m}(x, \theta)$ for fixed $x \in[0,1]$ and $\theta \in I$. Therefore, if $\lfloor c\rfloor \geq\lfloor m / 2\rfloor+1$, then $\theta_{m}(x)$ lies in $(-A, A)$ for all $x \in(0,1)$. It follows from the Comparison Theorem that $\theta_{1}(1)<\theta_{m}(1)$ and a contradiction with (9) is achieved. Indeed, if $\lfloor c\rfloor<\lfloor m / 2\rfloor+1$, then by (9) and Corollary 6 ,

$$
\lfloor c\rfloor \geq\left\lfloor\frac{\lfloor m / 2\rfloor \pi+\delta_{k \mu_{m}}}{\delta_{K \mu_{1}}}\right\rfloor=\left\lfloor\frac{\lfloor m / 2\rfloor \pi+\tan ^{-1}\left(c \tan \delta_{K \mu_{1}}\right)}{\delta_{K \mu_{1}}}\right\rfloor \geq\lfloor m / 2\rfloor+1,
$$

a contradiction. The proof of (a) is complete. 
To prove (b), we compare the differential equations $\theta_{j}^{\prime}(x)=F_{j}\left(x, \theta_{j}\right)(j=1, m)$ on $(0,1)$ with another set of initial values

It follows that

$$
\theta_{1}(0)=\frac{\gamma_{K \mu_{1}}-\pi}{\sqrt{K \mu_{1}}} \text { and } \theta_{m}(0)=\frac{\gamma_{k \mu_{m}}-\pi}{\sqrt{k \mu_{m}}}
$$

Assume

$$
\theta_{1}(1)=\frac{\delta_{K \mu_{1}}}{\sqrt{K \mu_{1}}} \text { and } \theta_{m}(1)=\frac{(m-1) \pi+\delta_{k \mu_{m}}}{\sqrt{k \mu_{m}}}
$$

$$
\frac{\mu_{m}}{\mu_{1}} \geq\left(\frac{(m-1) \pi+\delta_{k \mu_{m}}}{\delta_{K \mu_{1}}}\right)^{2} \frac{K}{k} \quad \text { for some } m>1 .
$$

Owing to Lemma $5, \theta_{1}(0)<\theta_{m}(0)$. Thus $\theta_{1}(1)<\theta_{m}(1)$ if $\lfloor c\rfloor \geq m$. Indeed, if $\lfloor c\rfloor<m$, by Corollary 6 and $(10)$,

$$
\lfloor c\rfloor \geq\left\lfloor\frac{(m-1) \pi+\delta_{k \mu_{m}}}{\delta_{K \mu_{1}}}\right\rfloor=\left\lfloor\frac{(m-1) \pi+\tan ^{-1}\left(c \tan \delta_{K \mu_{1}}\right)}{\delta_{K \mu_{1}}}\right\rfloor \geq m,
$$

a contradiction. Hence half of $(6)$ is proved. The transformation $x \longmapsto 1-x$ produces the rest. Therefore (b) is also valid.

Theorem 9. Suppose all the conditions in Theorem 8 are satisfied.

(a) If $\pi / 2 \leq \min \{\delta, \pi-\gamma\}$, then for all $m>n \geq 1$,

$$
\frac{\mu_{m}}{\mu_{n}} \leq\left(2\left\lfloor\frac{1}{2}\left\lceil\frac{m}{n}\right\rceil\right\rfloor+1\right)^{2} \frac{K}{k} .
$$

(b) If $\pi / 2>\max \{\delta, \pi-\gamma\}$, then for all $m>n>1$,

$$
\frac{\mu_{m}}{\mu_{n}}<\left(2\left\lceil\frac{m}{n}\right\rceil-1\right)^{2} \frac{K}{k} \text {. }
$$

Proof. We follow the method in [2]. First assume that $n$ divides $m$. Use mathematical induction in $n$. The case $n=1$ follows from Theorem 8. Suppose part (a) holds from $m=h n(h \in \mathbf{N})$. Fix $i \in N$; for each $j<i$, let $N_{j}\left(\mu_{i}\right)$ denote the point in $(0,1)$ such that the phase function of the $i$ th eigenfunction first attains $\pi / 2$ $(\bmod \pi)$ after $j$ zeros in $(0,1)$. Let $\omega_{1}=N_{1}\left(\mu_{n+1}\right)$ and $\omega_{2}=N_{h}\left(\mu_{h(n+1)}\right)$. If $\omega_{1} \leq \omega_{2}$, we consider the same regular Sturm-Liouville system on $\left(0, \omega_{1}\right)$. Now for each $h \in \mathbf{N}$, suppose $\widetilde{\mu_{h}}$ is the $h$ th eigenvalue. Then $\mu_{h(n+1)} \leq \widetilde{\mu_{h}}$. By Theorem 8 and Lemma 7,

$$
\frac{\mu_{h(n+1)}}{\mu_{n+1}} \leq \frac{\widetilde{\mu_{h}}}{\widetilde{\mu_{1}}} \leq\left(2\left\lfloor\frac{h}{2}\right\rfloor+1\right)^{2} \frac{K}{k} .
$$

On the other hand, if $\omega_{1}>\omega_{2}$, we perform the transformation $t=1-x$ and consider the regular Sturm-Liouville system on $\left(0,1-\omega_{1}\right)$ with a new separated boundary condition

$$
\begin{aligned}
y(0) \cos \delta-p(0) y^{\prime}(0) \sin \delta & =0, \\
y^{\prime}\left(1-\omega_{1}\right) & =0 .
\end{aligned}
$$

Therefore

$$
\frac{\mu_{h(n+1)}}{\mu_{n+1}} \leq \frac{\widetilde{\mu_{h n}}}{\widetilde{\mu_{n}}} \leq\left(2\left(\left\lfloor\frac{h}{2}\right\rfloor\right)+1\right)^{2} \frac{K}{k}
$$

by the induction hypothesis. In general let $h=\lceil m / n\rceil$. The proof for (b) is similar. 
Proof of Theorem 1. In view of Proposition 10 below, it suffices to consider the case when $0<\delta, \pi-\gamma<\pi / 2$. Here $\mu_{n}>0$ for $n \geq 2$. We apply the method in Theorem 9 to this case. Since the phase of one of the boundary points attains $\pi / 2$ $(\bmod \pi)$, Theorem 8 asserts that Eqs. (11) and (12) are still valid.

Proof of Theorem 2. The inequality follows from Theorems 8 and 9. When equality holds and $n=1$, then $\theta_{1}(1)=\theta_{m}(1)$. By the Comparison Theorem, $\theta_{1}(0)=\theta_{m}(0)$ and $F_{1}(x, \theta)=F_{m}(x, \theta)$. So $q \equiv 0$ and $k=K$. Moreover, since $\theta_{1}(x)=\theta_{m}(x)$ at $x=0,1$,

$$
\sqrt{\frac{k \mu_{m}}{K \mu_{1}}}=2\left\lfloor\frac{m+1}{2}\right\rfloor-1=2\left\lfloor\frac{m}{2}\right\rfloor+1,
$$

which implies that $m$ has to be odd. The converse is trivial. Then we use induction on $n$. The proof is similar to [2, Proposition 3.2] and will be omitted.

\section{Sign OF $\mu_{1}$ AND OTHER CASES}

While it is well known that when $q \geq 0, \mu_{2}$ is always positive (cf. [4, p. 318]), the sign of $\mu_{1}$ seems to be undetermined. In this section we show that when $q \geq 0$, $\mu_{1}$ is positive except possibly when $\delta, \pi-\gamma<\pi / 2$. In the latter case, positive $\mu_{1}$ is guaranteed when $q$ is sufficiently large. An upper estimate for $\mu_{m} / \mu_{1}$ immediately follows.

After the transformation (see [3])

$$
\frac{d t}{d x}=\frac{1}{p(x)}, \quad z(t)=y(x)
$$

the regular Sturm-Liouville system as given in (1)-(3) becomes another regular Sturm-Liouville system on $\left(0, t_{0}\right)$,

$$
-z^{\prime \prime}(t)+p(x(t)) q(x(t)) z(t)=\mu p(x(t)) w(x(t)) z(t),
$$

satisfying

$$
\left\{\begin{array}{c}
z(0) \cos \gamma-z^{\prime}(0) \sin \gamma=0 \\
z\left(t_{0}\right) \cos \delta-z^{\prime}\left(t_{0}\right) \sin \delta=0
\end{array}\right.
$$

where $t_{0}=\int_{0}^{1} p(x)^{-1} d x$. Applying classical Prüfer substitution on Eq. (13),

$$
z(t)=r(t) \sin \phi(t), \quad z^{\prime}(t)=r(t) \cos \phi(t),
$$

we have (cf. [4]) the following phase equation:

$$
\left\{\begin{aligned}
\phi^{\prime}(t) & =(\mu p w-p q) \sin ^{2} \phi(t)+\cos ^{2} \phi(t) \\
\phi(0) & =\gamma
\end{aligned}\right.
$$

If $\mu_{1} \leq 0$, then we compare Eqs. (13) and (14) with the differential equation (assuming $q \geq 0$ ),

$$
\hat{z}^{\prime \prime}=0,
$$

and its phase equation

$$
\left\{\begin{array}{l}
\hat{\phi}^{\prime}(t)=\cos ^{2} \hat{\phi}(t) \\
\hat{\phi}(0)=\gamma
\end{array}\right.
$$


Obviously Eq. (15) has a solution $\hat{z}(t)=c_{1} t+c_{2}$ where $c_{1}, c_{2}$ are constants satisfying

$$
c_{2} \cos \gamma-c_{1} \sin \gamma=0 .
$$

From the Comparison Theorem, $\phi(t) \leq \hat{\phi}(t)$ for all $t \geq 0$. We note that any solution of (14) has a zero in $(0,1)$ iff $\phi\left(t_{1}\right)=\pi$ for some $t_{1} \in\left(0, t_{0}\right)$. Thus $\hat{\phi}\left(t_{1}\right) \geq \pi$. But the linear function $\hat{z}(t)$ has a zero in $\left(0, t_{0}\right)$ iff $\left(c_{1} t_{0}+c_{2}\right) c_{2}<0$ or $\frac{c_{1}}{c_{2}} t_{0}+1<0$. Hence by $(16),-t_{0}<\tan \gamma<0$. The transformation $t \longmapsto t_{0}-t$ also gives $0<\tan \delta<t_{0}$. Thus we have proved the following proposition.

Proposition 10. If $q \geq 0$, then the eigenvalues $\mu_{1}$ of a regular Sturm-Liouville system is positive, except possibly when

$$
-\int_{0}^{1} p(x)^{-1} d x<\tan \gamma<0 \text { and } 0<\tan \delta<\int_{0}^{1} p(x)^{-1} d x
$$

which imply $0<\delta, \pi-\gamma<\pi / 2$.

Theorem 11. Suppose the regular Sturm-Liouville system (1)-(3) satisfies $0<$ $k \leq p w \leq K$. If $\varphi=\max \{\delta, \pi-\gamma\}<\pi / 2$ and $b \equiv \min _{[0,1]} p q>\cot ^{2} \varphi$, then $\mu_{1}>0$ and

$$
\frac{\mu_{m}}{\mu_{1}} \leq\left(m-\frac{1}{2}\right)^{2} \frac{\pi^{2} K}{\zeta^{2} k}
$$

where

$$
\zeta=\tan ^{-1}[b \tan \varphi-\cot \varphi] .
$$

Proof. In view of Theorem 8(b), we only need to prove that $\varphi_{K \mu_{1}} \geq \zeta$, i.e. $K \mu_{1}>$ $b-\cot ^{2} \varphi$. WLOG, let $\varphi=\pi-\gamma$. Hence it suffices to show that $K \mu_{1}>b-\cot ^{2} \gamma$. Let $\xi=K \mu_{1}-b$. We compare Eq. (13) with

$$
-\tilde{z}^{\prime \prime}=\xi \tilde{z} .
$$

Then the phase equation becomes

$$
\left\{\begin{array}{l}
\frac{d \tilde{\phi}}{d t}=\xi \sin ^{2} \tilde{\phi}(t)+\cos ^{2} \tilde{\phi}(t), \\
\phi(0)=\gamma>\pi / 2 .
\end{array}\right.
$$

So $\phi(t) \leq \tilde{\phi}(t)$ for all $t \geq 0$. If $\xi<\cot ^{2} \gamma$, then $\tilde{\phi}^{\prime}(t)<0$ whenever $\tilde{\phi}(t)=\gamma$. On the other hand, $\tilde{\phi}^{\prime}(t)>0$ whenever $\tilde{\phi}(t)=0$. It follows that $\tilde{\phi}(t)$ lies in $(0, \gamma]$ and never attains $\pi$, which means that $\tilde{z}$ has no zero in $(0, \infty)$. This result contradicts the fact that $z$ has a zero in $\left(0, t_{0}\right)$.

Our method of translating the phase function to the negative real axis works only when the eigenfunction has at least a zero in $(0,1)$. The method fails when we consider the zeroth eigenvalue $\mu_{0}$. However, if one boundary condition is a Dirichlet one (say, $\gamma=0$ ), then a straightforward use of the Ashbaugh-Benguria method would give

$$
\frac{\mu_{m}}{\mu_{1}} \leq\left(\frac{(m-1) \pi+\delta_{k \mu_{m}}}{\delta_{K \mu_{1}}}\right)^{2} \frac{K}{k}
$$

provided that $\mu_{1}>0$ and arrive at an explicit upper estimate when $\delta \geq \pi / 2$. 


\section{ACKNOWLEDGMENT}

We thank Tson-Tzer Lu and Chao-Liang Shen for introducing us to this area of study. We also thank Zi-Cai Li, Jhishen Tsay, and Ngai-Ching Wong for helpful and stimulating discussions.

\section{REFERENCES}

1. M. S. Ashbaugh and R. D. Benguria, Best constant for the ratio of the first two eigenvalues of one-dimensional Schrödinger operators with positive potentials, Proc. Amer. Math. Soc. 99 (1987) 598-599. MR 88e:34039

2. M. S. Ashbaugh and R. D. Benguria, Optimal bounds for ratios of eigenvalues of onedimensional Schrödinger operators with Dirichlet boundary conditions and positive potentials, Comm. Math. Phys. 124 (1989) 403-415. MR 91c:34114

3. M. S. Ashbaugh and R. D. Benguria, Eigenvalue ratios for Sturm-Liouville operators, J. Diff. Eqns. 103 (1993) 205-219. MR 94c:34125

4. G. Birkhoff and G. C. Rota, "Ordinary Differential Equations", 4th ed (1989) Wiley, New York. MR 90h:34001

Department of Applied Mathematics, National Sun Yat-Sen University, Kaohsiung, TAIWAN 80424, RePublic OF China

E-mail address: law@sun1.math.nsysu.edu.tw 